\title{
ON THE LOWER BOUND OF THE NUMBER OF REAL ROOTS OF A RANDOM ALGEBRAIC EQUATION WITH INFINITE VARIANCE
}

\author{
G. SAMAL AND M. N. MISHRA
}

ABstract. Let $N_{n}$ be the number of real roots of a random algebraic equation $\sum_{0}^{n} \xi_{v} x^{n}=0$, where the coefficients $\xi_{r}$ 's are independent random variables with common characteristic function exp $\left(-C|t|^{x}\right), C$ being a positive constant and $\alpha \geqq 1$. It is proved that

$$
N_{n} \geqq(\mu \log n) /(\log \log n) .
$$

The measure of the exceptional set tends tc zero as $n$ tends to infinity.

1. Introduction. Consider the algebraic equation

$$
f(x)=\xi_{0}+\xi_{1} x+\cdots+\xi_{n} x^{n}=0
$$

where the $\xi_{v}$ 's are independent random variables assuming real values only, and let $N_{n}$ be the number of real roots of (1).

The problem of finding a lower bound of $N_{n}$ has been considered by several authors. But none has taken up the case when the coefficients have infinite variance. Samal [3] has considered the general case when the $\xi_{v}$ s have identical distribution, with expectation zero and variance, and third absolute moment finite and nonzero.

(2) The object of this paper is to find a lower bound for $N_{n}$ when the $\xi_{v}$ 's are identically distributed with a common characteristic function $\exp \left(-C|t|^{\alpha}\right)$, where $C$ is a positive constant and $\alpha \geqq 1$. For $1 \leqq \alpha<2$ this represents a symmetric stable distribution with infinite variance.

A stronger result has been obtained by Littlewood and Offord [2] in the case $\alpha=2$. They have proved that $N_{n} \geqq(\mu \log n) /(\log \log \log n)$ except for a set of measure which tends to zero as $n$ tends to infinity.

Although our result is weaker than the result obtained by them, it holds for all $\alpha \geqq 1$. The impcrtance of our result lies in the range $1<\alpha<2$ when the variance is infinite.

Our main theorem is the following:

THEOREM. Let $f(x)$ be a polynomial of degree $n$ chosen at random with condition (2). Then for $n \geqq n_{0}$, the number of real roots of most of the equations

Received by the editors June 21, 1971.

AMS 1969 subject classifications. Primary 60XX, 6090.

Key words and phrases. Random variables, stable distribution, infinite variance, rand om algebraic equations.

(c) American Mathematical Society 1972 
$f(x)=0$ is at least $(\mu \log n) /(\log \log n)$. The measure of the exceptional set tends to zero as $n$ tends to infinity.

We shall always suppose that any inequality is satisfied when $n$ is large. We shall denote positive constants by $\mu$.

2. The following lemma is necessary in the beginning of the proof.

LEMMA 1. If a random variable $\xi(u)$ has characteristic function $\exp \left(-C|t|^{\alpha}\right)$ then

for every $\varepsilon>0$.

$$
\operatorname{Pr}(|\xi(u)|>\varepsilon)<\frac{2^{1+\alpha} \cdot C}{1+\alpha} \cdot \frac{1}{\varepsilon^{x}}
$$

Proof. Let $F(x)$ be the distribution function of $\xi(u)$.

Then, by Gnedenko and Kolmogorov [1, p. 53],

$$
\begin{aligned}
\operatorname{Pr}(|\xi(u)|>\varepsilon) & =1-\{F(\varepsilon)-F(-\varepsilon)\} \\
& \leqq 1-\left\{\frac{1}{2} \varepsilon\left|\int_{-2 / \varepsilon}^{2 / \varepsilon} \exp \left(-C|t|^{\alpha}\right) d t\right|-1\right\} \\
& =2-\frac{1}{2} \varepsilon \int_{-2 / \varepsilon}^{2 / \varepsilon} \exp \left(-C|t|^{\alpha}\right) d t \\
& =2-\varepsilon \int_{0}^{2 / \varepsilon} \exp \left(-C|t|^{\alpha}\right) d t \\
& \leqq 2-\varepsilon \int_{0}^{2 / \varepsilon}\left(1-C t^{\alpha}\right) \\
& =\frac{\varepsilon C}{1+\alpha}(2 / \varepsilon)^{1+\alpha}=\frac{2^{1+\alpha} \cdot C}{1+\alpha} \cdot \frac{1}{\varepsilon^{1+\alpha}} .
\end{aligned}
$$

3. Proof of the Theorem. We shall follow the line of proof of Samal [3] with modifications where necessary.

(3) Take constants $A$ and $B$ such that $0<B<1$ and $A>1$ and let $\lambda=$ $\log n$.

Let $M$ be the integer defined by

$$
M=\left[2^{1+x} A e \lambda^{\alpha} / B\right]+1
$$

where $[x]$ is the integral part of $x$; and let $k$ be determined by

$$
M^{2 k} \leqq n<M^{2 k+2} \text {. }
$$

We consider the polynomial $f=\sum_{v=0}^{n} \xi_{v} x^{v}$ at the points

$$
x_{m}=\left(1-\frac{1}{M^{2 m}}\right)^{1 / \alpha} \quad\left(m=\left[\frac{1}{2} k\right]+1,\left[\frac{1}{2} k\right]+2, \cdots, k\right) .
$$


There are $\frac{1}{2} k$ points if $k$ is even and $\frac{1}{2}(k+1)$ points if $k$ is odd. We have

$$
f\left(x_{m}\right)=U_{m}+R_{m}
$$

where

$$
U_{m}=\sum_{M^{2 m-1}+1}^{M^{2 m+1}} \xi_{v} x_{m}^{v} \text { and } R_{m}=\left(\sum_{0}^{M^{2 m-1}}+\sum_{M^{2 m+1}}^{n}\right) \xi_{v} x_{m}^{v} .
$$

Obviously $U_{m}$ and $U_{m+1}$ are independent random variables. We also define

$$
V_{m}=\left(\sum_{M^{2 m+1}+1}^{M^{2 m+1}} x_{m}^{\alpha v}\right)^{1 / \alpha}
$$

We proceed to estimate

$$
\begin{aligned}
P= & \operatorname{Pr}\left\{\left(U_{2 m}>V_{2 m}, U_{2 m+1}<-V_{2 m+1}\right) \cup\left(U_{2 m}<-V_{2 m}, U_{2 m+1}>V_{2 m+1}\right)\right\} \\
= & \operatorname{Pr}\left(U_{2 m}>V_{2 m}\right) \operatorname{Pr}\left(U_{2 m+1}<-V_{2 m+1}\right) \\
& +\operatorname{Pr}\left(U_{2 m}<-V_{2 m}\right) \operatorname{Pr}\left(U_{2 m+1}>V_{2 m+1}\right) .
\end{aligned}
$$

Since the charactersitic function of $\xi_{v}(u)$ is $\exp \left(-C|t|^{\alpha}\right)$ therefore the characteristic function of $U_{m}$ is

$$
\exp \left\{-C|t|^{\alpha} \sum_{M^{2 m-1}+1}^{M^{2 m+1}} x_{m}^{\alpha v}\right\}=\exp \left\{-C|t|^{\alpha} V_{m}^{\alpha}\right\}
$$

Hence the characteristic function of $U_{m} / V_{m}$ is $\exp \left\{-C|t|^{\alpha}\right\}$ which is independent of $m$.

If $F(x)$ is the distribution function of $U_{m l} / V_{m}$ for all $m$, then

$$
\begin{aligned}
P & =\{1-F(1)\} F(-1)+F(-1)\{1-F(1)\} \\
& =2 F(-1)\{1-F(1)\}=\delta \quad \text { (say). }
\end{aligned}
$$

Obviously $\delta>0$.

We define $E_{m}$ and $F_{m}$ as follows:

$$
\begin{aligned}
& E_{m}=\left\{U_{2 m}>V_{2 m}, U_{2 m+1}<-V_{2 m+1}\right\}, \\
& F_{m}=\left\{U_{2 m}<-V_{2 m}, U_{2 m+1}>V_{2 m+1}\right\} .
\end{aligned}
$$

We have shown above that $m\left(E_{m} \cup F_{m}\right)=\delta$.

Let $\eta_{m}$ be a random variable such that it takes value 1 on $E_{m} \cup F_{m}$ and zero elsewhere. In other words,

$$
\begin{aligned}
\eta_{m} & =1 \quad \text { with probabiiity } \delta, \\
& =0 \quad \text { with probability } 1-\delta .
\end{aligned}
$$

It follows as in Samal [3, p. 439] that outside a set of measure at most $\mu / k$, either $U_{2 m}>V_{2 m}$ and $U_{2 m+1}<-V_{2 m+1}$ or $U_{2 m}<-V_{2 m}$ and $U_{2 m+1}>$ $V_{2 m+1}$ for at least $\mu k$ values of $m$. 
4. Before we consider $R_{m}$ we need the following lemmas.

LEMMA 2.

$$
\left|\sum_{M^{2 m+1}+1}^{n} \xi_{v}(u) x_{m}^{v}\right|<\frac{1}{2} V_{m}
$$

except for a set of measure at most $\left\{2^{1+2 \alpha} \cdot C /(1+\alpha)\right\}(A e / B) e^{-M}$.

PROOF. The characteristic function of $\sum_{M^{2 m+1+1}}^{n} \xi_{v}(u) x_{m}^{v}$ is

$$
\exp \left\{-C|t|^{\alpha} \sum_{M^{2 m+1+1}}^{n} x_{m}^{\alpha v}\right\} .
$$

Hence by using Lemma 1 ,

$$
\begin{aligned}
P_{1} & =\operatorname{Pr}\left\{\left|\sum_{M^{2 m+1}+1}^{n} \xi_{v}(u) x_{m}^{v}\right| \geqq \frac{1}{2} V_{m}\right\} \\
& \leqq\left\{2^{1+\alpha} \cdot C \sum_{M^{2 m+1}+1}^{n} x_{m}^{\alpha v}\right\} /(1+\alpha)\left(\frac{1}{2} V_{m}\right)^{\alpha} \\
& =\left(C \cdot 2^{1+2 \alpha} \sum_{M^{2 m+1}+1}^{n} x_{m}^{\alpha v}\right) /(1+\alpha) V_{m}^{\alpha} .
\end{aligned}
$$

But

$$
\begin{aligned}
\sum_{M^{2 m+1}+1}^{n} x_{m}^{\alpha v} & \leqq \frac{x_{m}^{\alpha}\left(M^{2 m+1}+1\right)}{1-x_{m}^{\alpha}}=M^{2 m}\left(1-\frac{1}{M^{2 m}}\right)^{M^{2 m+1}+1} \\
& \leqq M^{2 m}\left(1-\frac{1}{M^{2 m}}\right)^{M^{2 m+1}+1} \\
& \leqq M^{2 m}\left(1-\frac{1}{M^{2 m}}\right)^{M^{2 m+1}} \leqq M^{2 m} e^{-M}
\end{aligned}
$$

Also it follows as in Samal [3, p. 439] that

$$
\begin{aligned}
V_{m}^{\alpha} & =\sum_{M^{2 m-1}+1}^{M^{2 m+1}} x_{m}^{\alpha v} \geqq \sum_{M^{2 m-1}+1}^{M^{2 m}} x_{m}^{\alpha v} \\
& \geqq\left(M^{2 m}-M^{2 m-1}\right)\left(1-\frac{1}{M^{2 m}}\right)^{M^{2 m}} \geqq M^{2 m} B \cdot \frac{e^{-1}}{A} .
\end{aligned}
$$

Therefore

$$
\begin{aligned}
P_{1} & \leqq \frac{C \cdot 2^{1+2 \alpha}}{1+\alpha} \frac{M^{2 m} e^{-M}}{M^{2 m} B\left(e^{-1} / A\right)} \\
& =\frac{2^{1+2 \alpha} \cdot C}{1+\alpha} \frac{A e}{B} e^{-M}
\end{aligned}
$$


Lemma 3.

$$
\left|\sum_{0}^{M^{2 m-1}} \xi_{v}(u) x_{m}^{v}\right|<\lambda\left(\sum_{0}^{M^{2 m-1}} x_{m}^{\alpha v}\right)^{1 / \alpha}
$$

except for a set of measure at most $2^{1+\alpha} \cdot C /(1+\alpha) \lambda^{\alpha}$.

Proof. The characteristic function of $\sum_{0}^{M^{2 m-1}} \xi_{v}(u) x_{m}^{v}$ is

$$
\exp \left\{-C|t|^{\alpha} \sum_{0}^{M{ }^{2 m-1}} x_{m}^{\alpha v}\right\}
$$

Therefore, by using Lemma 1,

$$
P_{2}=\operatorname{Pr}\left\{|| \sum_{0}^{M^{2 m-1}} \xi_{v}(u) x_{m}^{v} \mid \geqq \lambda\left(\sum_{0}^{M^{2 m-1}} x_{m}^{\alpha v}\right)^{1 / \alpha}\right\} \leqq 2^{1+\alpha} \cdot C /(1+\alpha) \lambda^{\alpha} .
$$

Thus by using Lemmas 2 and 3, we have, for any given $m$,

$$
\left|R_{m}\right|<\frac{1}{2} V_{m}+\lambda\left(\sum_{0}^{M^{2 m-1}} x_{m}^{\alpha v}\right)^{1 / \alpha}
$$

except for a set of measure at most $\mu_{1} e^{-I I}+\mu_{2} / \lambda^{x}$ where $\mu_{1}$ and $\mu_{2}$ are positive constants which can be determined in terms of the initial constants $C, \alpha, A$ and $B$. But

$$
\begin{aligned}
\lambda\left(\sum_{0}^{M^{2 m-1}} x_{m}^{\alpha v}\right)^{1 / \alpha} & <\lambda\left(M^{2 m-1}+1\right)^{1 / \alpha} \\
& \leqq \lambda M^{(2 m-1) / \alpha}\left(1+\frac{1}{M^{2 m-1}}\right)^{1 / \alpha} \\
& \leqq 2^{1 / \alpha} \lambda M^{(2 m-1) / \alpha} \\
& =2^{1 / \alpha} \lambda M^{2 m / \alpha} / M^{1 / \alpha} \\
& \leqq 2^{1 / \alpha} \lambda\left(\frac{A e}{B}\right)^{1 / \alpha} V_{m} / M^{1 / \alpha} \\
& =\lambda\left(\frac{2 A e}{B M}\right)^{1 / \alpha} V_{m} .
\end{aligned}
$$

The last steps above follow from (10). Hence by using (4), it follows that

$$
\left|R_{m}\right|<V_{m}
$$

for $m=\left[\frac{1}{2} k\right]+1,\left[\frac{1}{2} k\right]+2, \cdots, k$. Thus it follows from (7) that except for a set of measure at most

$$
(\mu / k)+\mu_{1} \cdot \frac{1}{2}(k+1) e^{-M}+\mu_{2} \cdot \frac{1}{2}(k+1) / \lambda^{\alpha} .
$$


$f$ shows a change of sign and therefore has a root, between $x_{2 m}$ and $x_{2 m+1}$ for at least $\mu k$ values of $m$.

It can be easily shown by using (4) and (5) that

and

$$
\mu_{1}(\log n)^{x} \leqq M \leqq \mu_{2}(\log n)^{x}
$$

$$
\left(\mu_{3} \log n\right) /(\log \log n) \leqq k \leqq\left(\mu_{4} \log n\right) /(\log \log n) .
$$

We shall complete the proof of the theorem by showing that the measure of the exceptional set tends to zero as $n$ tends to infinity.

$$
\begin{aligned}
k e^{-M} & \leqq\left\{\left(\mu_{4} \log n\right) /(\log \log n)\right\} \exp \left\{-\mu_{1}(\log n)^{\alpha}\right\} \\
& =\left(\mu_{1} / \log \log n\right) \cdot\left\{(\log n) / \exp \left[\mu_{1}(\log n)^{\alpha}\right]\right\}
\end{aligned}
$$

which tends to zero since $\alpha \geqq 1$. Again

$$
\begin{aligned}
k / \hat{\lambda}^{\alpha} & \leqq\left\{\left(\mu_{4} \log n\right) /(\log \log n)\right\} /(\log n)^{\alpha} \\
& =\left\{\mu_{4} /(\log \log n)\right\} /(\log n)^{\alpha-1}
\end{aligned}
$$

which tends to zero for the same reason as above. Also $1 / k$ tends to zero as $\eta$ tends to infinity. Hence (11) tends to zero as $n$ tends to infinity. Obviously, the measure of the exceptional set is $O\left(1 /(\log \log n)(\log n)^{\alpha-1}\right)$ if $1 \leqq \alpha<2$ and $O(\log \log n / \log n)$ if $\alpha \geqq 2$, when $n$ tends to infinity.

\section{REFERENCES}

1. B. V. Gnedenko and A. N. Kolmogorov, Limit distributions for sums of independent random variables, GITTL, Moscow, 1949; English transl., Addison-Wesley, Reading, Mass., 1954. MR 12, 839; MR 16, 52.

2. J. E. Littlewood and A. C. Offord, On the number of real roots of a random algebraic equation. II, Proc. Cambridge Philos. Soc. 35 (1939), 133-148.

3. G. Samal, On the number of real roots of a random algebraic equation, Proc. Cambridge Philos. Soc. 58 (1962), 433-442. MR 25 \#2657.

Department of Mathematics, Ravenshaw College, Cuttack 3, Orissa, India

Bureau of Statistics, Cuttack, India 